\title{
Sensing metabolites using donor-acceptor nanodistributions in fluorescence resonance energy transfer
}

\author{
O. J. Rolinskia) and D. J. S. Birch \\ University of Strathclyde, Department of Physics and Applied Physics, John Anderson Building, \\ 107 Rottenrow, Glasgow G4 ONG, United Kingdom \\ L. J. McCartney and J. C. Pickup \\ Department of Chemical Pathology, Guy's King's and St. Thomas' School of Medicine, Guy's Hospital, \\ London SE1 9RT, United Kingdom
}

(Received 22 August 2000; accepted for publication 5 March 2001)

\begin{abstract}
Before fluorescence sensing techniques can be applied to media as delicate and complicated as human tissue, an adequate interpretation of the measured observables is required, i.e., an inverse problem needs to be solved. Recently we have solved the inverse problem relating to the kinetics of fluorescence resonance energy transfer (FRET), which clears the way for the determination of the donor-acceptor distribution function in FRET assays. In this letter this approach to monitoring metabolic processes is highlighted and the application to glucose sensing demonstrated. (C) 2001 American Institute of Physics. [DOI: 10.1063/1.1367308]
\end{abstract}

One of the strategic goals of clinical medicine is the rapid and noninvasive measurement of metabolic processes. Research into collisional quenching of fluorescence as a simple mechanism for detecting critical analytes has been extensive, e.g., blood oxygen. ${ }^{1}$ However, in many instances changes in metabolism are accompanied by subtle changes in macromolecular conformation which are difficult to reveal with simple concentration assays. Such diagnostics frequently present an inverse problem which reverses the typical, forward solution process, where all system inputs, sources and parameters are known and mathematical models are used to predict outputs and states. The inverse approach is particularly useful when the physical environment makes it difficult, impossible, or prohibitively expensive to directly measure the relevant physical parameters. Blood glucose level control in diabetic patients is a typical example, as the currently used methods require a blood sample to be taken from the patient and this must be followed by a chemical reaction in vitro. The global scale of diabetes (150 million people worldwide) has led to diverse research into in vivo glucose sensing, ${ }^{2}$ including fluorescence based approaches. ${ }^{2-5}$ In the latter, the level of analyte has to be determined from changes in a fluorescence characteristic of the fluorophore (e.g., mean lifetime), although hitherto no theory has been available to model such complex kinetics.

Among several molecular mechanisms which can be applied to the detection of an analyte or monitor a process in complex media, fluorescence resonance energy transfer (FRET) provides quite specific site (and hence structural) information. This is because the rate of FRET, $w(r)$

$$
w(r)=\left(1 / \tau_{0}\right)\left(R_{0} / r\right)^{6}
$$

depends strongly on the donor/acceptor distance $r$. The term $\tau_{0}$ is the donor fluorescence lifetime in the absence of FRET, and $R_{0}$ is the critical transfer distance.

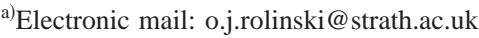

In the presence of an acceptor the donor fluorescence impulse response function $I_{D}(t)$ modifies from a monoexponential function $\exp \left(-t / \tau_{0}\right)$ to the form ${ }^{6}$

$$
I_{D}(t)=\exp \left[-\frac{t}{\tau_{0}}-\int_{0}^{\infty} d r \rho(r)(1-\exp [-t w(r)])\right] .
$$

Here $\rho(r)$ is the donor-acceptor distribution function, where $\int_{0}^{R} \rho(r) d r$ is the number of acceptor molecules in a volume of a sphere of radius $R$.

In this letter we concentrate on the inverse problem constituted by the Eq. (2) in which the donor-acceptor distribution function $\rho(r)$ is an input information which is to be recovered from the output signal $I_{D}(t)$. Of course, the transmitted information might be modified during metabolic sensing by the detection method applied, tissue autofluorescence and tissue scatter, however these tissue-specific effects are not discussed here. The recognition mechanism involved (e.g., competitive binding) establishes a relationship between analyte concentration $[A]$ and $\rho(r)$. In the detection stage $\rho(r)$ is converted into $I_{D}(t)$ according to Eq. (2). This detection mechanism does not transfer the full original information into measurable fluorescence characteristics, thus some information is lost (just like during a telephone conversation where the higher frequencies are attenuated), and results in no possibility of the full recovery of $\rho(r)$ from the donor fluorescence decay $I_{D}(t)$. However, as we have recently shown, a distribution sensing approach, ${ }^{7}$ adding another dimension to the experiment by taking a series of measurements of $I_{D}(\alpha ; t)$ for the different values of the parameter $\alpha=R_{0}^{6} / \tau_{0}$, enables the complete picture of $\rho(r)$ to be built up. The determination of the $\rho(r ;[A])$ at the distance range $\sim 5-100 \AA$ would be extremely advantageous for numerous biomedical sensing applications, fluorescence decay measurements with ns resolution being all that are required. ${ }^{8}$

Problems similar to that posed by Eq. (2) arise in a wide variety of biomedical applications, and consist of using mathematical models in order to determine unknown system 


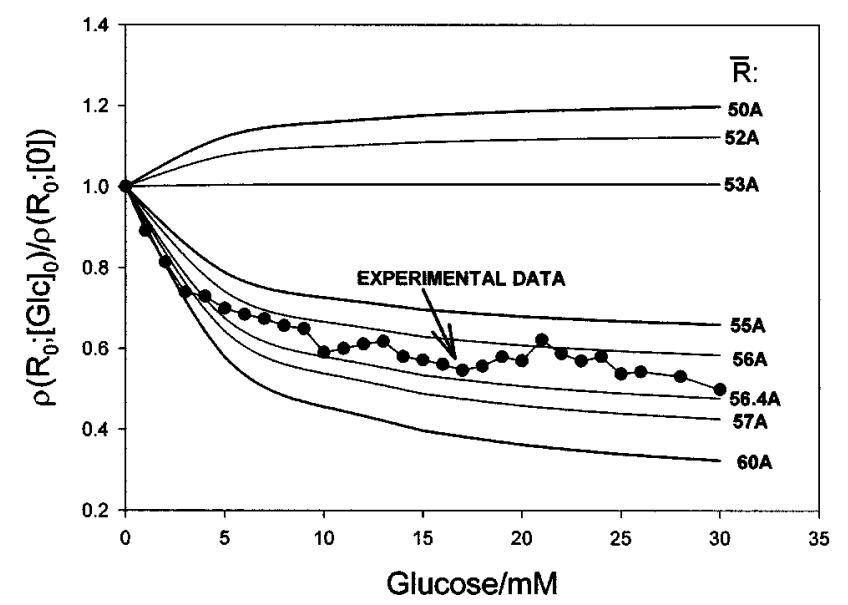

FIG. 1. APC/MG distribution function at the distance $R_{0}=59.8 \AA$, $\rho\left(59.8 \AA ;[\mathrm{Glc}]_{0}\right) / \rho(59.8 \AA ; 0)$, compared with the simulated curves obtained from a model distribution function [Eq. (6)] for a series of average donor-acceptor distances $\bar{R}$. The experimental values of $[\mathrm{Dex}]_{0}$ $=6.68 \mu \mathrm{M}$ and $[\mathrm{ConA}]_{0}=16.7 \mu \mathrm{M}$ and the arbitrary chosen $n=p=15$ were used in simulations.

inputs, sources or parameters from observed system outputs and responses. A common approach is to use a mathematical model to fit, often using least-square error analysis, predictions of the model to the measured system outputs by adjusting the unknown model parameters. For example, the integral in Eq. (2) is usually solved for the assumed random three- or two-dimensional distribution $\rho(r)$ and the parameter $\gamma,{ }^{9}$ indicating the acceptor concentration, is determined from the experimental data. This simple approach is not appropriate to systems where the process of interest (e.g., conformational change, competitive binding, etc.) is accompanied by a change in $\rho(r)$. FRET based distribution sensing ${ }^{7}$ makes no a priori assumption regarding the donor-acceptor distribution function and is presented here applied to an affinity sensor for glucose. In this widely researched type of sensor, $^{3-5}$ a highly fluorescent donor dye [in our case the phycobiliprotein, allophycocyanin (APC)] and a nonfluorescent acceptor, e.g., in our case malachite green (MG) isothiocyanate are used. Change in the APC/MG distribution function detected by FRET to a MG-Dextran complex from APC bound to glucose binding protein Concanavalin A (ConA) due to displacement of the complex by glucose from ConA provides the basis of the assay. We demonstrate that even data which use only one $R_{0}$ value, are capable of offering structural information. Hence we suggest that distribution sensing will find much wider application in studying structure and dynamics on the nanometer scale, for example, in proteins, membranes, polymers, and amorphous solids.

The donor-acceptor distribution function $\rho(r)>0$ is expressed in our approach as the infinite series of the orthonormal Laguerre polynomials $L_{k}^{s}(r)$ with the coefficients $a_{k}^{(s)}$ without sacrificing generality of the function $\rho(r)$

$$
\rho(r)=r^{s} \sum_{k=0}^{\infty} a_{k}^{(s)} L_{k}^{s}(r),
$$

where $s$ is an arbitrary chosen parameter. Combining Eqs. (3) and (2) and then applying to both sides of the resulting expression the operator

Fression the operator
Downloaded 21 Apr 2010 to 130.159.17.136. Redistribution subject to AlP license or copyright; see http://apl.aip.org/apl/copyright.jsp

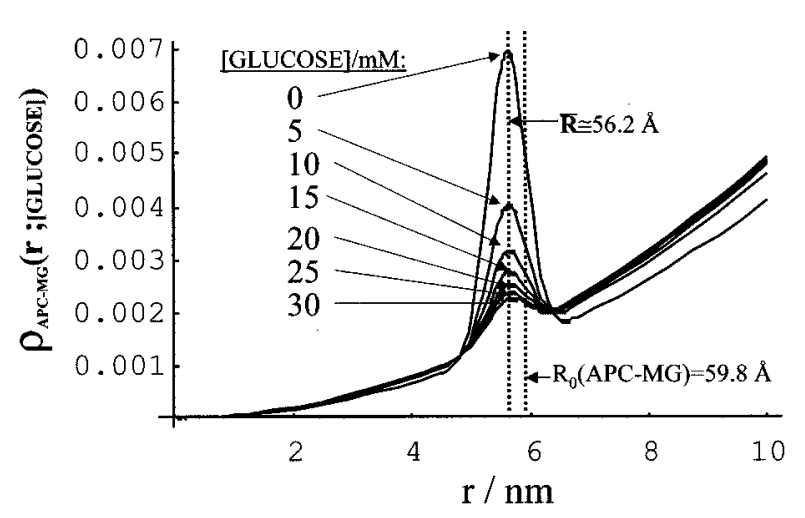

FIG. 2. The complete APC/MG distribution function for different glucose levels $\rho\left(r ;[\mathrm{Glc}]_{0}\right)$ in an APC-ConA:Dextran-MG complex, recovered by the method of distribution sensing.

$$
\int_{0}^{\infty} d t e^{-t} L_{p}(t)^{*}
$$

defined in time space, we obtain

$$
f_{\alpha, p}=\sum_{k=0}^{m} a_{k}^{(s)} b_{k p}^{(s)}(\alpha) .
$$

Equation (5) establishes the fundamental relationship between the experimentally available vector $\mathbf{f}_{\alpha}=\left\{f_{\alpha, 0}\right.$, $\left.f_{\alpha, 1}, f_{\alpha, 2}, \ldots, f_{\alpha, p}\right\}$ defined in the time domain, the sought for vector $\mathbf{a}^{(s)}=\left\{a_{0}^{s}, a_{1}^{s}, a_{2}^{s}, \ldots, a_{M}^{s}\right\}$ defined in the distance domain, and the matrix $\mathbf{b}^{(s)}(\alpha)$, which depends on $\alpha=R_{0}^{6} / \tau_{0}$ only. The basic procedure for determining of $\rho(r)$ in this approach assumes several steps. In the first step the parameter $\alpha$ is estimated from the steady-state $\left(R_{0}\right)$ and timeresolved $\left(\tau_{0}\right)$ measurements. Next, the donor-acceptor system for which $\rho(r)$ is to be determined is prepared, the donor fluorescence decay is measured and the $\mathbf{f}$ vector calculated. In the next step the mode of representation of the donoracceptor distribution function ( $s$ value) has to be chosen and the matrix $\mathbf{b}^{(s)}(\alpha)$ calculated. Finally, vector $\mathbf{a}^{(s)}$ is found from solving the set of Eq. (5) and $\rho(r)$ is calculated on the basis of Eq. (4). The donor/acceptor pair of particular $R_{0}$

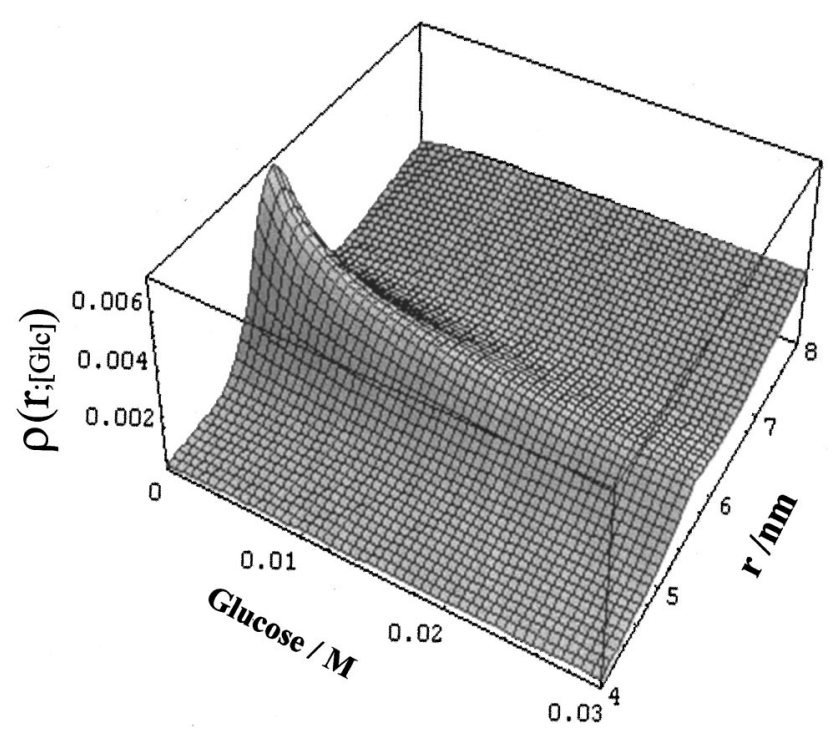


value reports only the value of $\rho(r)$ in the narrow region $\left(R_{0}-d R_{0}, R_{0}+d R_{0}\right){ }^{7}$ Thus, improved accuracy of $\rho(r)$ requires the measurements to be made over a range of $R_{0}$ values.

The glucose affinity sensor under consideration consists of five types of species, namely the APC-labeled ConA (receptor), the MG-labeled Dextran, glucose and two complexes: APC-ConA:Dextran-MG and APC-ConA:Glucose. The changes in APC/MG distribution function at the distance $R_{0}=59.8 \AA, \rho\left(59.8 \AA\right.$; $\left.[\mathrm{Glc}]_{0}\right)$, has been determined experimentally for glucose concentrations, $[\mathrm{Glc}]_{0}$, from 0 to 30 $\mathrm{mM}$ which covers the physiological range. The coefficients $a_{k}^{(1 / 2)}$ for $k=0,1,2, \ldots, 15$ were found numerically by solving a set of Eq. (5) and then Eq. (3) was used to determine $\rho\left(59.8 \AA ;[\mathrm{Glc}]_{0}\right)$. The matrix elements $b_{k p}^{(s)}(\alpha)$ were calcu- lated using $R_{0}=59.8 \AA$ and $\tau_{0}=1.90 \mathrm{~ns}$ (measured separately) for $\alpha$ determination. In order to determine vector $\mathbf{f}_{\alpha}$, the digitized donor fluorescence decays $I_{D}(t)$, determined for the samples of different glucose concentrations $[\mathrm{Glc}]_{0}$, were recovered from the experimental time-resolved data using a fast Fourier transform.

Figure 1 shows the APC/MG distribution function at the distance $R_{0}=59.8 \AA, \rho\left(59.8 \AA ;[\mathrm{Glc}]_{0}\right)$, normalized with respect to the value, $\rho(59.8 \AA ; 0)$, namely $\rho(59.8 \AA$; $\left.[\mathrm{Glc}]_{0}\right) / \rho(59.8 \AA ; 0)$. This is compared with the simulated curves obtained from a model distribution function for a series of average donor-acceptor distances in a complex $\bar{R}$. The model glucose controlled donor-acceptor distribution function of the form

$$
\rho\left(r ;[\mathrm{Glc}]_{0}\right)=4 \pi[\mathrm{Dex}] N_{A} r^{2}+\left\{\begin{array}{l}
\frac{4 \pi N_{A}}{3} \frac{\Gamma(n+p+2)}{\Gamma(p+1) \Gamma(n+1)}(2 \bar{R})^{2-n-p}[\operatorname{ConADex}] r^{n}(2 \bar{R}-r)^{p}, \quad 0 \leqslant r \leqslant 2 \bar{R} \\
0, \quad r>2 \bar{R}
\end{array}\right.
$$

has been used in this analysis to provide interpretation to the experimental data. Factors $4 \pi N_{A}$ and $\left(4 \pi N_{A} / 3\right)(\Gamma(n+p$ $\left.+2) /[\Gamma(p+1) \Gamma(n+1)](2 \bar{R})^{2-n-p}\right)$ ensure normalization of $\rho\left(r ;[\mathrm{Glc}]_{0}\right)$. The term $\sim r^{2}$ corresponds to the randomly distributed (unbound) MG-Dextran molecules, while the expression $\sim r^{n}(2 \bar{R}-r)^{p}$ describes distribution of MG molecules around an APC molecule in an APCConA:Dextran-MG complex. The $\rho\left(r ;[\mathrm{Glc}]_{0}\right)$ depends parametrically on the glucose level (as [Dex] and [ConADex $]$ are controlled by $[\mathrm{Glc}]_{0}$ ) and on the APCConA:Dextran-MG morphology, represented here by $\bar{R}$.

The good agreement between the experimental data and theoretical curves validates the appropriateness of the Eq. (6) and provides an estimate of $\bar{R}$ in the APCConA:Dextran-MG complex to be $\sim 56.2 \AA$. Thus, it is now possible to demonstrate a complete $\rho\left(r ;[\mathrm{Glc}]_{0}\right)$ function and analyze how it is affected by $[\mathrm{Glc}]_{0}$ (Fig. 2). It becomes clear that the largest change in APC-MG distribution occurs for the initial small amounts of glucose $(0-5 \mathrm{mM})$, while for the higher glucose levels $(5-30 \mathrm{mM})$ the overall change is less. The sensor sensitivity $\left(\partial \rho\left(R_{0},[\mathrm{Glc}]_{0}\right) / \partial[\mathrm{Glc}]_{0}\right)$ would be higher for $R_{0}$ closer to $\bar{R}$ than what is actually observed. On the other hand, if $R_{0}$ was less than $50 \AA$ or more than 65 $\AA$, there would be no change or a very slight increase in $\rho\left(R_{0} ;[\mathrm{Glc}]_{0}\right) / \rho\left(R_{0} ; 0\right)$ on the appearance of glucose. This effect is illustrated better on a three-dimensional plot, Fig. 3, presenting a full $\rho\left(r ;[\mathrm{Glc}]_{0}\right)$ function. If $R_{0}$ is properly chosen $\left(R_{0} \simeq \bar{R}\right)$, the sensing parameter moves along a hill with glucose level increase, resulting in the greatest dynamic range of the assay.

The estimated average donor-acceptor distance $\bar{R}$ $\cong 56.2 \AA$ is consistent with crystallographic studies, ${ }^{10}$ which have shown that APC molecules consist of identical monomers assembled into trimers which form disk-like structures. The trimer has a radius of $\sim 55 \AA$ and a thickness $\sim 30 \AA$ with a central channel of $\sim 35 \AA$ diameter. Each monomer Downloaded 21 Apr 2010 to 130.159 .17 .136 . Redistribution subject possesses an internal fluorophore $\left(1 \alpha 84^{10}\right)$, and when an APC-ConA:Dextran-MG complex is formed, it is very likely that an internal fluorophore-MG distance lies in the range corresponding to the estimated value for $\bar{R}$ of $56.2 \AA$.

The application of the distribution sensing reported here demonstrated its ability to recover structural information on nanometer systems even for limited experimental data (e.g., one $R_{0}$ value). Fluorescence decay measurements for a series of $R_{0}$ values enable determination of $\rho(r)$ in the required region of distances and with required accuracy. Then, by applying prior information on the system, the relation between $\rho(r)$ and the analyte or process of interest can be established. This might enable the wider application of distribution sensing, not just in medical diagnostics, but also in biochemistry and materials science.

The authors wish to acknowledge research grants from EPSRC and The Wellcome Trust.

${ }^{1}$ O. S. Wolfbeis, in Biomedical Optical Instrumentation and Laser-Assisted Biotechnology, edited by V. Scheggi (Kluwer, Dordrecht, 1996).

${ }^{2}$ For a recent review, see J. Pickup, L. McCartney, O. Rolinski and D. Birch, BMJ 319, 1289 (1999).

${ }^{3}$ O. J. Rolinski, D. J. S. Birch, L. J. McCartney, and J. C. Pickup, J. Photochem. Photobiol., B 54, 26 (2000).

${ }^{4}$ L. Tolosa, H. Szmacinski, G. Rao, and J. R. Lakowicz, Anal. Biochem. 250, 102 (1997).

${ }^{5}$ L. Tolosa, H. Malak, G. Rao, and J. R. Lakowicz, Sens. Actuators B 45, 93 (1997).

${ }^{6}$ J. Klafter, J. M. Drake and A. Blumen, in Kinetics and Catalysis in Microheterogeneous Systems, edited by M. Grätzel and K. Kalyanasundaram (Dekker, New York, 1991), Chap. 14

${ }^{7}$ O. J. Rolinski and D. J. S. Birch, J. Chem. Phys. 112, 8923 (2000).

${ }^{8}$ D. J. S. Birch and R. E. Imhof, Topics in Fluorescence Spectroscopy, edited by J. R. Lakowicz (Plenum, New York, 1991), Vol. 1, Chap. 1.

${ }^{9}$ See, for example, O. J. Rolinski and D. J. S. Birch, Meas. Sci. Technol. 10, 127 (1999).

${ }^{10}$ G. N. Reeke, Jr., J. W. Becker, and G. N. Edelman, J. Biol. Chem. 250, 4 (1975).

AIP license or copyright; see http://apl.aip.org/apl/copyright.jsp 